\title{
Universal thermodynamics of strongly interacting Fermi gases
}

\author{
Hui Hu, ${ }^{1,2}$ Peter D. Drummond,, , and Xia-Ji Liu ${ }^{2}$ \\ ${ }^{1}$ Department of Physics, Renmin University of China, Beijing 100872,China \\ ${ }^{2}$ ARC Centre of Excellence for Quantum-Atom Optics, Department of Physics, \\ University of Queensland, Brisbane, Queensland 4072, Australia
}

(Dated: June 21, 2018)

Strongly interacting Fermi gases are of great current interest. Not only are fermions the most common particles in the universe, but they are also thought to have a universal thermodynamic behavior for strong interactions [1, 2, 3]. Recent experiments on ultra-cold Fermi gases provide an unprecedented opportunity to test universality in the laboratory $[4,5,6,7,8]$. In principle this allows - for example - the interior properties of hot, dense neutron stars to be investigated on earth. Here we carry out a detailed test of this prediction. We analyze results from three ultra-cold fermion experiments involving two completely distinct atomic species in different kinds of atomic trap environments [6, 7, 8]. The data is compared with the predictions of a recent strong interaction theory [9, 10]. Excellent agreement is obtained, with no adjustable parameters. By extrapolating to zero temperature, we show that the experimental measurements yield a many-body parameter $\beta \simeq-0.59 \pm .07$, describing the universal energy of strongly interacting Fermi gases.

Experiments on ultra-cold Fermi gases at micro-Kelvin temperatures are revolutionizing many areas of physics. Their exceptional simplicity allows tests of many-body theory in areas long thought to be inaccessible. The ability to widely tune the effective interaction between fermions via a broad Feshbach resonance in gases of ${ }^{6} \mathrm{Li}$ and ${ }^{40} \mathrm{~K}$ has allowed resonance models proposed in highTc superconductivity theory 11 to be implemented with fermionic ultra-cold atoms [12]. Fortunately, the Pauli exclusion principle stabilizes the resulting excited molecular states against collisional damping [13]. This has permitted the experimental observation of the smooth evolution of the Fermi gas from the attractive regime of Bardeen-Cooper-Schrieffer (BCS) superfluidity through to a regime where molecules form a Bose-Einstein condensate (BEC) [14, 15, 16, 17, 18, 19]. On the cusp of this BCS-BEC crossover, there is a strongly interacting regime - the so-called unitarity limit [3], which leaves the inter-atomic distance as the only relevant length scale.

At this point, the gas is expected to exhibit a universal thermodynamic behaviour, independent of any microscopic details of the underlying interactions. The ground state energy $E_{0}$ of a homogeneous gas at zero tempera- ture should be proportional to the free Fermi energy, $E_{F}$. Thus, $E_{0}=E_{F}(1+\beta)$, where $\beta$ is a universal many-body parameter. Substantial experimental efforts have been carried out to verify the existence of universality [4, [5], though so far there has been no conclusive confirmation. This is mainly due to the lack of reliable thermometry in the strongly interacting regime [10], leading to an uncertainty in the finite temperature corrections.

The universal parameters estimated from energies at the lowest accessible (but unknown) temperature, range from $\beta=-.68 \pm 0.1$ to $\beta=-.54 \pm 0.05[6,6$,, 16 , 17. Since these are not at zero temperature, there is an unknown correction factor required to obtain the ground state energy, and hence the true value of $\beta$. There is a similar range of estimated theoretical values, though a more precise value of $\beta=-0.58 \pm 0.01$ was recently obtained from zero temperature quantum Monte Carlo simulations [20].

This situation has dramatically improved in the most recent thermodynamic measurements on strongly interacting Fermi gases of ${ }^{40} \mathrm{~K}$ and ${ }^{6} \mathrm{Li}$ atoms [7, 8], which allow accurate estimates of the energy in the universal regime from the fermionic cloud size. In experiments on ${ }^{40} \mathrm{~K}$ carried out at JILA [7], an adiabatic magnetic field sweep is used to compare measurements in the strongly interacting and weakly interacting regimes, so that the non-interacting temperature is also known from the cloud size after the sweep. An important conceptual advance of the Duke group [5] who use ${ }^{6} \mathrm{Li}$, was the realization that this gives a model-independent measurement of the entropy in the strongly-interacting regime, thus allowing a precision test [8] of theoretical predictions of universal thermodynamics. A different approach at Rice [6], also with ${ }^{6} \mathrm{Li}$, makes use of the detailed density distribution to estimate temperature and entropy.

These ground-breaking investigations provide measurements accurate to the level of a few percent, which is an exceptional accuracy in this challenging field of ultralow temperature physics.

In this Letter, we re-analyze all the available experimental data from these three laboratories, thus obtaining the measured entropy-energy relation of two completely different strongly interacting trapped Fermi gases. We compare this directly with a single universal theoretical prediction. We use a diagrammatic approach based on functional path-integrals [9, 21] together with the local density approximation to treat the inhomoge- 


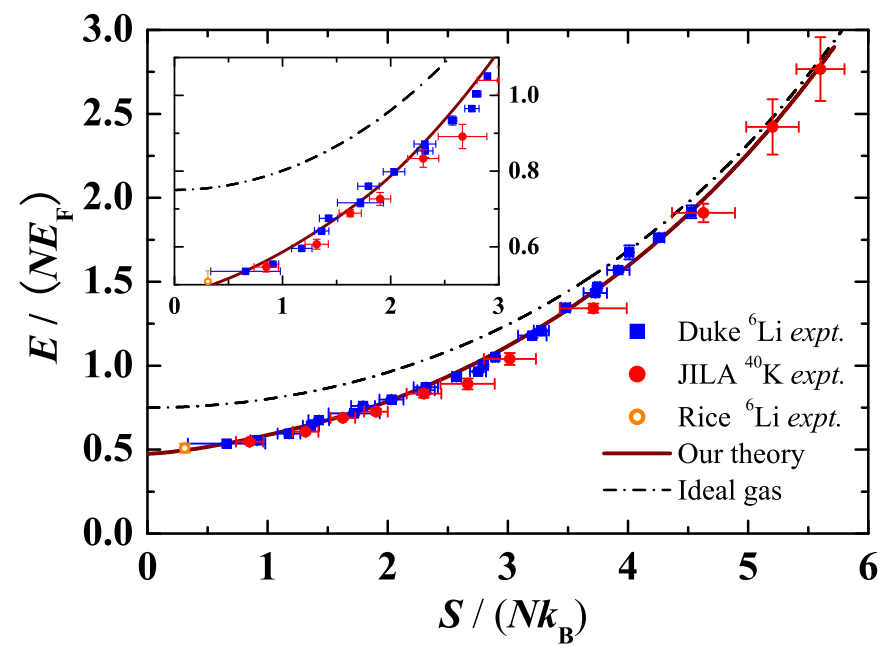

Figure 1: (Color online) Illustration of the universal thermodynamics of a strongly interacting Fermi gas. Comparison between theoretical predictions and experimental measurements on the entropy-energy relation of strongly interacting Fermi gases in a harmonic trap. Inset highlights the low entropy region. The energy is in units of the Fermi energy, which is the highest single-particle energy level of a non-interacting gas in the same trap with the same number of fermions, $N$. Brown solid curve is our theoretical prediction, and the black dash-dotted curve is the ideal gas energy. The experimental data is from Refs. [6, 7] and [8], on ${ }^{6} \mathrm{Li}$ and ${ }^{40} \mathrm{~K}$ fermionic atoms, with error-bars taken from the experimental papers using appropriate conversions. All results agree with a single, universal theoretical curve.

neous trap. Below the superfluid transition, our calculations include pairing fluctuations, which are important in the strongly interacting regime, due to the onset of molecule formation. This approach is described in detail elsewhere [9]. Above threshold we use the well-known Nozières-Schmidt-Rink (NSR) theory [22, 23, 24]. We demonstrate a quantitative test of these thermodynamic predictions by comparing experimental results on both types of atom with a single theoretical curve, as shown in Fig (1). There are no adjustable parameters, so this provides strong evidence for universality.

Further, by using power-law extrapolation to estimate finite temperature corrections, we are able to show that all the experimental data give rise to a universal manybody coefficient of $\beta \simeq-0.59 \pm 0.07$. This is in excellent agreement both with the Monte Carlo results [20], $\beta=$ $-0.58 \pm 0.01$, and our earlier theoretical prediction [9] of $\beta \approx-0.599$.

We summarize the experimental procedures, as typified by the JILA work using ${ }^{40} \mathrm{~K}$ atomic gases [7]. Here, the strongly interacting gas is prepared in a harmonic trap at the Feshbach resonance, and the potential energy is measured from the observed radius. Next, the magnetic field is swept adiabatically to a zero scattering length field, and the potential energy at this field is again measured, which gives the non-interacting temperature. From this data, we obtain the entropy of the interacting gas [7], since the energy-entropy relation of a non-interacting Fermi gas is known. The total energy is also obtained, as it is twice the potential energy at the resonance, owing to the virial theorem [5]. Fig. 2a gives the predicted potential energy ratio as a function of the non-interacting temperature in the presence of a harmonic trap, together with the experimental measurements. The observed reduction of the potential energy in the strongly interacting regime is theoretically reproduced. Converting the non-interacting temperature and the potential energy into the total entropy and energy, respectively, we obtain the entropy-energy relation for ${ }^{40} \mathrm{~K}$ gas in Fig. 2b. We find an excellent agreement between the experimental data and theoretical predictions below threshold. There is a small discrepancy just above the critical temperature of $\left(T / T_{F}\right)^{0} \approx 0.25$ or the critical entropy of $S_{c} \simeq 2.2 N k_{B}$, where we expect that the above threshold NSR theory may be less reliable. This effect is clearly visible in Fig. 2a, which gives the original experimental measurements. The nonlinear transformation used to obtain the entropy-energy relation in Fig. $2 \mathrm{~b}$ means that conventional rectangular error-bars give only a qualitative indication of the uncertainties in this figure.

Next, we discuss in greater detail the ${ }^{6} \mathrm{Li}$ experiment in Duke [8], which has greater accuracy but involves some extra corrections due to the anharmonic trap used, and residual interactions in the weakly interacting cloud. The strongly interacting Fermi gas of $N=1.3(2) \times 10^{5}$ atoms is prepared in a shallow Gaussian (anharmonic) trap $V(\mathbf{r})=U_{0}\left\{1-\exp \left[-m\left(\omega_{\perp}^{2} \rho^{2}+\omega_{z}^{2} z^{2}\right) /\left(2 U_{0}\right)\right]\right\}$ at a magnetic field $B=840 \mathrm{G}$, slightly above the resonance position $B_{0}=834 \mathrm{G}$. The coupling constant $k_{F} a=-30.0$, where $k_{F}$ is the Fermi wave vector and $a$ is the $s$-wave inter-atomic scattering length, is sufficient large to ensure the onset of the universal thermodynamic behavior. Experimentally, the entropy of the gas is measured by an adiabatic passage to a weak interacting field $B=1200 \mathrm{G}$, where $k_{F} a=-0.75$ and the entropy and temperature is known from the cloud size after the sweep. The energy $E$ is determined model independently from the mean square radius of the strongly interacting fermion cloud $\left\langle z^{2}\right\rangle_{840}$ measured at $840 \mathrm{G}$, according to the virial theorem [ [5,, 8$]$,

$$
\frac{E}{N E_{F}}=\frac{\left\langle z^{2}\right\rangle_{840}}{z_{F}^{2}}(1-\kappa),
$$

where $E_{F}=\left(3 N \omega_{\perp}^{2} \omega_{z}\right)^{1 / 3}=k_{B} T_{F}$ is the Fermi energy for an ideal harmonically trapped gas at the trap center, and $z_{F}^{2}$ is defined by $3 m \omega_{z}^{2} z_{F}^{2} \equiv E_{F}$. The correction factor $1-\kappa$ accounts for the anharmonicity in the shallow trapping potential $U_{0} \simeq 10 E_{F}$.

Fig. 3a shows the bare experimental data on the ratio of the mean square axial cloud size at $1200 \mathrm{G}$ to that at 

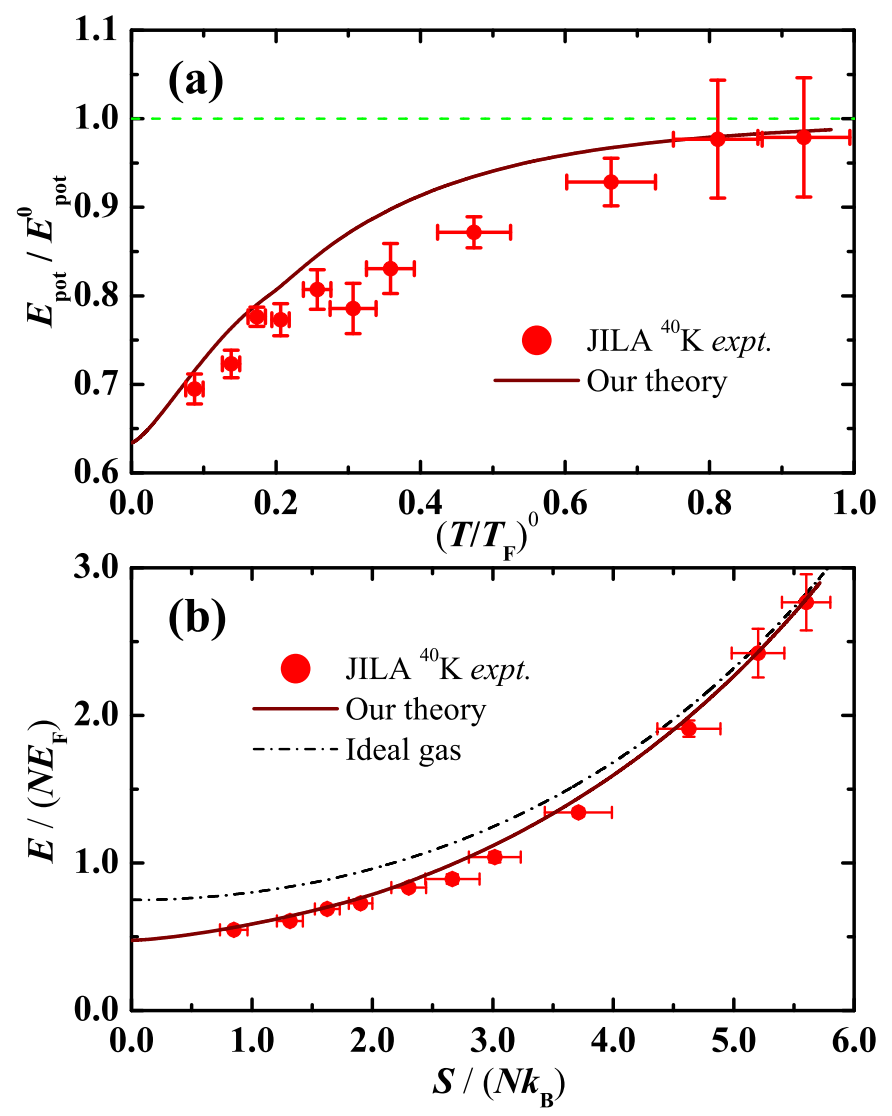

Figure 2: (Color online) Analysis of the ${ }^{40} \mathbf{K}$ experiment in JILA. a, Experimental data on the potential energy $E_{p o t}$ normalized to that in the non-interacting regime $E_{p o t}^{0}$ for a ${ }^{40} \mathrm{~K}$ gas at unitarity, as a function of the non-interacting gas temperature $\left(T / T_{F}\right)^{0}$. It converges to unity as expected (dashed green horizontal line). The experimental data are compared with our theoretical prediction for a harmonically trapped, strongly interacting Fermi gas. b, Comparison between theory and experiment on the entropy dependence of the energy for a unitarity gas. The experimental entropy is calculated from the non-interacting temperature, with errorbars converted from the experimental data. The energy is double the potential energy $E_{\text {pot }}$ due to the virial theorem [5].

$840 \mathrm{G}$, as a function of the energy at $840 \mathrm{G}$, as compared to the theoretical simulations with the same realistic parameters, except that we use a resonance field $B_{0}$ for the strongly interacting gas. Good agreement is found, with no free parameters. As before, there is a small discrepancy between the raw data and theoretical predictions, just above the critical energy. We have recalculated the entropy corrections due to residual interactions in the $1200 \mathrm{G}$ cloud to improve the accuracy at the $1 \%$ level, by using an above-threshold NSR theory. Calibration of the entropy from the measured mean square axial cloud size at $1200 \mathrm{G}$ using the theoretically predicted dependence of the entropy on the size (inset in Fig. 3b) leads to the comparison for the entropy-energy relation, as shown in Fig. $3 \mathrm{~b}$. The agreement is even more impressive.
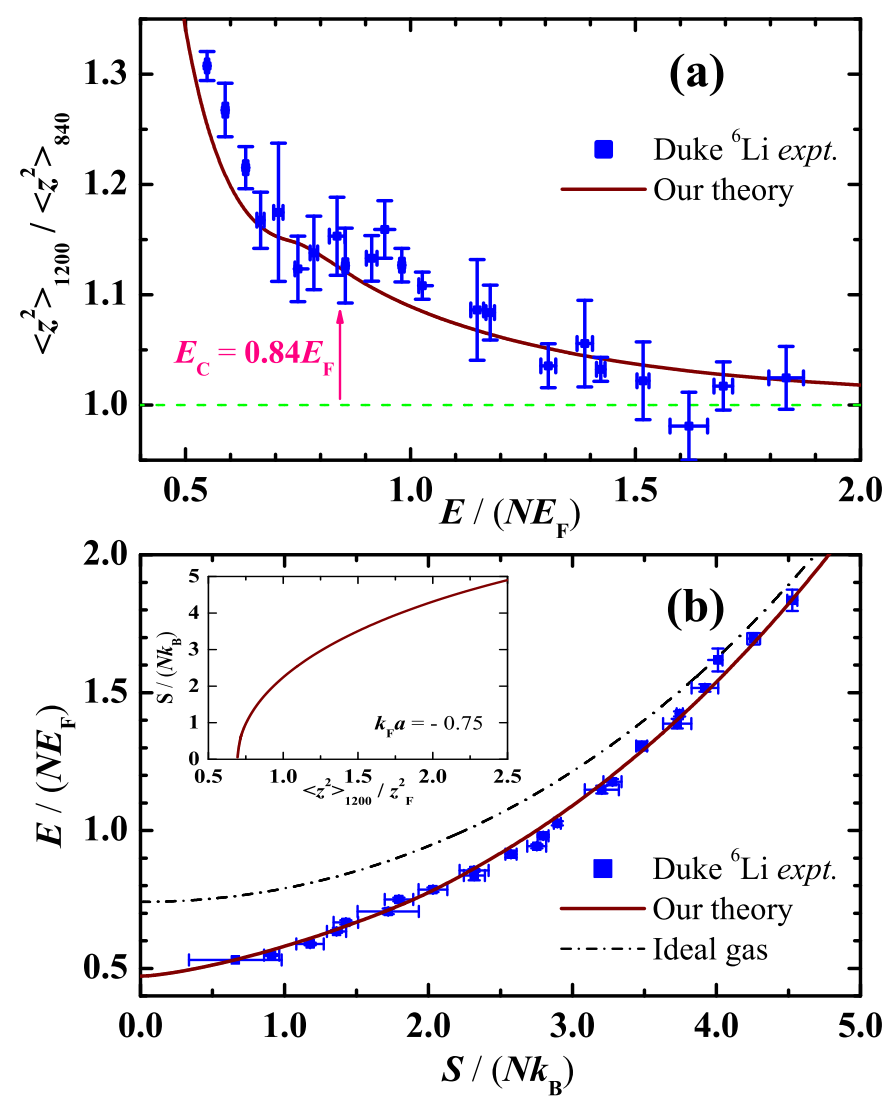

Figure 3: (Color online) Analysis of the ${ }^{6} \mathbf{L i}$ experiment in Duke. a, Experimental data on the ratio of the mean square cloud size at $1200 \mathrm{G},\left\langle z^{2}\right\rangle_{1200}$ to that at $840 \mathrm{G},\left\langle z^{2}\right\rangle_{840}$ for a ${ }^{6} \mathrm{Li}$ gas, as a function of total energy, are compared to the theoretical simulations. The data is obtained by an adiabatic passage from a strongly interacting field $B=840 \mathrm{G}$ to a weakly interacting field $B=1200 \mathrm{G}$. The total energy is measured at $840 \mathrm{G}$ by utilizing the virial theorem, and is normalized with respect to the Fermi energy of a non-interacting gas. The theoretical curve are calculated under the same procedure and parameters, except that the starting field is at the Feshbach resonance $B=834 \mathrm{G}$. As shown by the dashed green horizontal line, the ratio converges to unity at high energy as the gas becomes more ideal. The arrow points to the theoretically predicted energy at transition point. b, Experimental data on the entropy dependence of the energy for a strongly interacting gas at $840 \mathrm{G}$, compared to the predictions from our strong interaction theory. The result for an ideal gas is also plotted. The entropy and error-bars of the gas are calibrated from the experimental mean square axial cloud size $\left\langle z^{2}\right\rangle_{1200}$, using our theoretical dependence of the entropy on the cloud size (as shown in the inset), which should be extremely accurate in the weakly interacting regime.

We can now describe the procedures used to obtain Fig (1), which illustrates the universal thermodynamic behavior of a strongly interacting Fermi gas. Here we have plotted all the measured data in a single figure, and compared them with our prediction for the entropy dependence of the energy of a harmonically trapped, strongly 
interacting Fermi gas, as well as that of an ideal Fermi gas. The slight shift of the experimental data in the Duke experiment due to the anharmonicity of the trap has been corrected, by subtracting the (small) theoretical difference between a shallow Gaussian trap and a harmonic trap for the energy at the same entropy. We plot also a single data point from the ${ }^{6} \mathrm{Li}$ experiment in Rice [6] for the energy at their lowest entropy. The agreement between theory and experiment is excellent for almost all the measured data. Exactly the same theory is used in all cases, with results from three different laboratories [6, 7, 8]. The universal thermodynamics of a strongly interacting Fermi gas is strikingly demonstrated, independently of which atomic species we compare with.

Just above the critical entropy $S_{c} \simeq 2.2 N k_{B}$, for the superfluid-normal fluid phase transition, there is a suggestion of a discrepancy between theoretical predictions and these precise measurements. At this point the abovethreshold NSR theory is least accurate [10]. The data here may even indicate a first order superfluid phase transition. We note that the exact order of phase transition for a strongly interacting Fermi gas is still an open question [25], and merits further investigation.

A key feature of current ultra-cold Fermi gas experiments is that the lowest attainable entropy is around $S=0.7 N k_{B}$, which corresponds to a temperature of $0.10-0.15 T_{F}$ at unitarity. This nonzero entropy or temperature affects the precise determination of the universal many-body parameter $\beta$. To remove the temperature dependence, we assume that in the low entropy regime (below the phase transition), there is a power law dependence of the energy on the entropy: $E-E_{0} \propto S^{\alpha}$, as anticipated theoretically. For non-interacting Fermi and Bose gases, the power law exponents are 2 and $4 / 3$, respectively. The thermodynamics of a unitary gas should lie between an ideal Fermi gas and an ideal Bose-Einstein condensate. The fitting procedure leads to $E_{0} /\left(N E_{F}\right)=0.48 \pm 0.03$ and $E_{0} /\left(N E_{F}\right)=0.48 \pm 0.04$, for the Duke and JILA experiments, respectively, with a similar power law exponent $\alpha=1.7 \pm 0.4$. Here the error bar accounts for the fitting error only. Using the relation $E_{0} / N=(3 / 4)(1+\beta)^{1 / 2} E_{F}$ for a harmonic trap [4], this gives rise to $\beta \simeq-0.59 \pm 0.07$ : which agrees fairly well with the most accurate quantum Monte Carlo simulations 20], $\beta=-0.58 \pm 0.01$, and our theoretical predictions [9], $\beta \approx-0.599$. Our theoretical power-law prediction is $\alpha=1.5$ in the LDA regime, which also agrees with experiment.

We are extremely grateful to J. E. Thomas and B. Clancy for many helpful discussions, and for sharing their data before publication. We also thank D. S. Jin and J. T. Stewart for communications on their data, and R. G. Hulet et. al. for explaining their temperature measurements. This work was supported by an Australian Research Council Center of Excellence grant, the National Natural Science Foundation of China Grant No. NSFC-
10574080, and the National Fundamental Research Program Grant No. 2006CB921404 and 2006CB921306. Correspondence and requests for materials should be addressed to P. D. D.

Methods. - We briefly explain our analytic theory. This is an approximate method using perturbation theory summed to all orders, since no exact results are known. In the homogeneous gas case, it relies on the many-body $T$-matrix approximation to account for the effects of collective Bogoliubov-Anderson modes, and extends the standard NSR approach to the brokensymmetry state [9]. This amounts to considering the contributions of Gaussian fluctuations around the meanfield saddle point to the thermodynamic potential (with Nambu notation),

$$
\delta \Omega=\frac{1}{2} \sum_{Q} \ln \operatorname{det}\left[\begin{array}{cc}
\chi_{11}(Q) & \chi_{12}(Q) \\
\chi_{12}(Q) & \chi_{11}(-Q)
\end{array}\right],
$$

where

$$
\begin{aligned}
\chi_{11}(Q) & =\frac{m}{4 \pi \hbar^{2} a}+\sum_{K} \mathcal{G}_{11}(Q-K) \mathcal{G}_{11}(K)-\sum_{\mathbf{k}} \frac{1}{2 \epsilon_{\mathbf{k}}}, \\
\chi_{12}(Q) & =\sum_{K} \mathcal{G}_{12}(Q-K) \mathcal{G}_{12}(K),
\end{aligned}
$$

are respectively the diagonal and off-diagonal parts of the pair propagator. Here, $Q=\left(\mathbf{q}, i \nu_{n}\right), K=\left(\mathbf{k}, i \omega_{m}\right)$, and $\sum_{K}=k_{B} T \sum_{m} \sum_{\mathbf{k}}\left(\mathbf{q}\right.$ and $\mathbf{k}$ are wave vectors, $\nu_{n}$ and $\omega_{m}$ bosonic and fermionic Matsubara frequencies, respectively), $m$ is the fermion mass, $T$ the temperature, and $\epsilon_{\mathbf{k}}=\hbar^{2} \mathbf{k}^{2} / 2 m, \mathcal{G}_{11}$ and $\mathcal{G}_{12}$ are BCS Green's functions with a variational order parameter $\Delta$. Together with the mean-field contribution

$$
\Omega_{0}=\sum_{\mathbf{k}}\left[\epsilon_{\mathbf{k}}-\mu+\frac{\Delta^{2}}{2 \epsilon_{\mathbf{k}}}+2 k_{B} T f\left(-E_{\mathbf{k}}\right)\right]-\frac{m \Delta^{2}}{4 \pi \hbar^{2} a},
$$

where the excitation energy $E_{\mathbf{k}}=\left[\left(\epsilon_{\mathbf{k}}-\mu\right)^{2}+\Delta^{2}\right]^{1 / 2}$ and the Fermi distribution function $f(x)=1 /\left(1+e^{x / k_{B} T}\right)$, we obtain the full thermodynamic potential $\Omega=\Omega_{0}+\delta \Omega$. All the observables are calculated straightforwardly following the thermodynamic relations, once the chemical potential $\mu$ and the order parameter $\Delta$ are determined. Number conservation in the form $n=-\partial \Omega / \partial \mu$ is strictly satisfied, yielding an exact identity for the pressure $P$ and energy density $\mathcal{E}$ of a unitarity gas: $P=2 / 3 \mathcal{E}[3,5]$. For simplicity, in our calculations we determine the order parameter at the level of mean field, using the gap equation $\partial \Omega_{0} / \partial \Delta=0$. Part of our approach was also previously derived using a functional integral method [21]. In the case of the normal Fermi liquid with vanishing order parameter, the usual NSR formalism is recovered [22].

This type of perturbation theory with bare BCS Green functions in the pair propagators constitutes the simplest description of the BCS-BEC crossover, including the essential pair fluctuations. More sophisticated approximations with dressed Green functions in the pair propagators, i.e., the $G G_{0}$ [26] and the fully self-consistent $G G$ 
schemes [27, 28], have also been proposed. In Ref. [10], we performed a comparative study of these different approximation schemes for a unitarity gas in the normal state. A related calculation in the superfluid phase has also been carried out recently [25]. Compared with the latest path-integral Monte Carlo simulations [29, 30], our analytic perturbation scheme seems to be the optimal choice for the calculations of the type required for the entropy-energy relation. However, there is a small region around the critical temperature where none of the current calculations are reliable, and we see indications of this in the data.

To include the effects of the trap, we employ the local density approximation by assuming that the system can be treated as locally uniform, with a position dependent local chemical potential $\mu(\mathbf{r})=\mu-V(\mathbf{r})$, where $V(\mathbf{r})$ is the trapping potential. The local entropy and energy, calculated directly from the local thermodynamic potential using thermodynamic relations, are then summed to give the total entropy and energy. We note that in the presence of a harmonic trap, the exact identity $P=2 / 3 \mathcal{E}$ yields the virial theorem [5], which states that the potential energy of the gas is a half of its total energy.

* Electronic address: drummond@physics.uq.edu.au

[1] Heiselberg, H. Fermi systems with long scattering lengths. Phys. Rev. A 63, 043606 (2001).

[2] Carlson, J., Chang, S.-Y., Pandharipande, V. R. \& Schmidt, K. E. Superfluid Fermi gases with large scattering length. Phys. Rev. Lett. 91, 050401 (2003).

[3] Ho, T.-L. Universal thermodynamics of degenerate quantum gases in the unitarity limit. Phys. Rev. Lett. 92, 090402 (2004).

[4] O’Hara, K. M., Hemmer, S. L., Gehm, M. E., Granade, S. R. \& Thomas, J. E. Observation of a strongly interacting degenerate Fermi gas of atoms. Science 298, 2179-2182 (2002).

[5] Thomas, J. E., Turlapov, A. \& Kinast, J. Virial theorem and universality in a unitary Fermi gas. Phys. Rev. Lett. 95, 120402 (2005).

[6] Partridge, G. B., Li, W., Kamar, R. I., Liao, Y.\& Hulet, R. G. Pairing and phase separation in a polarized Fermi gas. Science 311, 503-505 (2006).

[7] Stewart, J. T., Gaebler, J. P., Regal, C. A. \& Jin, D. S. Potential energy of a ${ }^{40} \mathrm{~K}$ Fermi gas in the BCS-BEC crossover. Phys. Rev. Lett. 97, 220406 (2006).

[8] Luo, L., Clancy, B., Joseph, J., Kinast, J. \& Thomas, J. E. Measurement of the entropy and critical temperature of a strongly interacting Fermi gas. Phys. Rev. Lett. 98, 080402 (2007).

[9] Hu, H., Liu, X.-J. \& Drummond, P. D. Equation of state of a superfluid Fermi gas in the BCS-BEC crossover. Europhys. Lett. 74, 574-580 (2006).

[10] Hu, H., Liu, X.-J. \& Drummond, P. D. Temperature of a trapped unitary Fermi gas at finite entropy. Phys. Rev. A 73, 023617 (2006).

[11] Friedberg, R. \& Lee, T. D. Gap energy and long-range order in the boson-fermion model of superconductivity. textitPhys. Rev. B 40, 6745-6762 (1989).

[12] Kheruntsyan, K. V. \& Drummond, P. D. Multidimensional quantum solitons with nondegenerate parametric interactions: photonic and Bose-Einstein condensate environments. Phys. Rev. A 61, 063816 (2000).

[13] Petrov, D. S., Salomon, C., \& Shlyapnikov, G. V. Weakly Bound Dimers of Fermionic Atoms. Phys. Rev. Lett. 93, 090404 (2004).

[14] Leggett, A. J. Modern Trends in the Theory of Condensed Matter (Springer-Verlag, Berlin, 1980).

[15] Regal, C. A., Greiner, M. \& Jin, D. S. Observation of resonance condensation of fermionic atom pairs. Phys. Rev. Lett. 92, 040403 (2004).

[16] Bourdel, T., Khaykovich, L., Cubizolles, J., Zhang, J., Chevy, F., Teichmann, M., Tarruell, L., Kokkelmans, S. J. J. M. F. \& Salomon C. Experimental study of the BEC-BCS crossover region in lithium 6. Phys. Rev. Lett. 93, 050401 (2004).

[17] Bartenstein, M., Altmeyer, A., Riedl, S., Jochim, S., Chin, C., Denschlag, J. Hecker \& Grimm, R. Crossover from a molecular Bose-Einstein condensate to a degenerate Fermi gas. Phys. Rev. Lett. 92, 120401 (2004).

[18] Zwierlein, M. W., Abo-Shaeer, J. R., Schirotzek, A., Schunck, C. H. \& Ketterle W. Vortices and superfluidity in a strongly interacting Fermi gas. Nature 435, 10471051 (2005).

[19] Romans, M. W. J. \& Stoof, H. T. C. Dressed Feshbach molecules in the BEC-BCS crossover. Phys. Rev. Lett. 95, 260407 (2005).

[20] Astrakharchik, G. E., Boronat, J., Casulleras, J. \& Giorgini, S. Equation of state of a Fermi gas in the BECBCS crossover: A quantum Monte Carlo study. Phys. Rev. Lett. 93, 200404 (2004).

[21] Engelbrecht, J. R., Randeria M. \& Sáde Melo, C. A. R. BCS to Bose crossover: Broken-symmetry state. Phys. Rev. B 55, 15153-15156 (1997).

[22] Nozières, P. \& Schmitt-Rink, S. Bose condensation in an attractive fermion gas: From weak to strong coupling superconductivity. J. Low Temp. Phys. 59, 195-211 (1985).

[23] Ohashi, Y. \& Griffin, A. BCS-BEC crossover in a gas of Fermi atoms with a Feshbach resonance. Phys. Rev. Lett. 89, 130402 (2002).

[24] Liu, X.-J. \& Hu, H. BCS-BEC crossover in an asymmetric two-component Fermi gas. Europhys. Lett. 75, 364-370 (2006).

[25] Haussmann, R., Rantner, W., Cerrito, S. \& Zwerger, W. Thermodynamics of the BCS-BEC crossover. Phys. Rev. A 75, 023610 (2007).

[26] Chen, Q., Stajic, J., Tan, S. N. \& Levin, K. BCS-BEC crossover: From high temperature superconductors to ultracold superfluids. Phys. Rep. 412, 1-88 (2005).

[27] Haussmann, R. Properties of a Fermi liquid at the superfluid transition in the crossover region between BCS superconductivity and Bose-Einstein condensation. Phys. Rev. B 49, 12975-12983 (1994).

[28] Liu, X.-J. \& Hu, H. Self-consistent theory of atomic Fermi gases with a Feshbach resonance at the superfluid transition. Phys. Rev. A 72, 063613 (2005).

[29] Bulgac, A., Drut, J. E. \& Magierski, P. Spin 1/2 fermions in the unitary regime: A superfluid of a new type. Phys. Rev. Lett. 96, 090404 (2006).

[30] Burovski, E., Prokof'ev, N., Svistunov, B. \& Troyer, M. Critical temperature and thermodynamics of attractive 
fermions at unitarity. Phys. Rev. Lett. 96, 160402 (2006). 